\title{
Análisis sobre nuevos modelos de relación en el desarrollo territorial y la gobernanza.Una mirada a las comarcas de Gipuzkoa ${ }^{1}$
}

http://dx.doi.org/10.15304/rips.18.1.4559

\author{
Andere Ormazabal Gaston, Izaro Gorostidi Vidaurrazaga, \\ Mario Zubiaga Garate \\ UNIVERSIDAD DEL PAÍS VASCO (UPV-EHU)
}

\begin{abstract}
Resumen: La reflexión contemporánea sobre la ordenación territorial hay que contextualizarla dentro de las circunstancias globales. El desarrollo de las comarcas y la gobernanza relacionada a ellas no pueden entenderse sin tener en cuenta los nuevos ajustes entre el capital, el territorio y la ciudadanía. El presente artículo se centra en el análisis de los modos de actuación para el desarrollo de las comarcas de Gipuzkoa. En concreto, analizaremos el proceso de gobernanza y la participación de los diferentes agentes dentro de la estrategia RIS3. Esta mirada global puede situar el debate ha realizar sobre modelos de desarrollo en el País Vasco, Gipuzkoa y sus comarcas.
\end{abstract}

Palabras clave: desarrollo territorial, gobernanza, especialización inteligente, RIS3, Gipuzkoa.

Abstract: The contemporary reflection about the territorial organization needs to situate in the global circumstance. The governance associated with the development of the regions can not be understood without paying attention to the new assembly among the capital, territory and the citizens. In this article we focus on the analysis of the modes of action in the development of the regions of Gipuzkoa. Specifically we will look at the governance process and the participation of actors in the RIS3 strategy.This general look can situate the discussion necessary to do about the development models in the Basque Country, in Gipuzkoa and in the regions of our territory.

Key words: territorial development, governance, smart specialization, RIS3, Gipuzkoa.

\section{Introducción}

1 primer cambio ideológico ha llegado en las últimas décadas, impulsado por el propio proceso de globalización y la aceleración de los subprocesos que ello conlleva. Las vías emprendidas son contradictorias y en Euskal Herria el desarrollo territorial también se ha situado en esa conflictiva coyuntura: las principales decisiones relacionadas con la competitivad se han alejado del ámbito ciudadano, y

1. Este artículo es el resultado de un proyecto de investigación financiado por la Diputación Foral de Gipuzkoa (Ikuskaritza Taldea GFA 2014). 
a su vez, como reacción, la ciudadanía reivindica su poder de decisión, impulsando procesos de democratización (Zubiaga, 2012).

En este momento, la aceleración del proceso de modernización viene acompañada de un salto tecnológico. La comprensión clásica de las escalas territoriales se ha quebrado. Como en la mayoría de otras veces, el nuevo equilibrio entre espacio y tiempo genera dudas y ante ellas nos aferramos a elementos supuestamente fijos: el pasado -o sea, la memoria histórica-, la tierra y el territorio. La única manera de recuperar la capacidad de tomar decisiones parece estar en situarse, esto es, prestando atención a lo local. Por eso, este tipo de construcción política no atiende únicamente a las variables ciudadanía/derechos y poder/autoridad, pues implica a su vez la reflexión sobre el territorio, y en nuestro caso, incluyendo las tres, el pueblo, nuevos modos de entender el propio sujeto colectivo.

En esa reflexión hay que tomar en cuenta diferentes variables: el hecho de dar centralidad a la innovación tecnológica y al conocimiento (Castells, 2000) y las nuevas escalas de medición de la competitividad exigen nuevas gobernanzas compartidas, orientadas a permitir una adecuada localización del territorio dentro de los mercados globales. Por otro lado, en la medida en que se ha consolidado la costrucción democrática, se ha reforzado la visión holística del desarrollo humano, poniendo en duda el mero crecimiento economicista. Al hilo de la consulta protegida mencionada por Tilly - ciudadanía más general e igualitaria, elección democrática de los mandatarios y control de la arbitrariedad (Tilly, 2004) -, se ha reivindicado la necesidad de extender el parámetro clásico de concentración relacionado a la separación entre estado social y capital también al ámbito de la regeneración del capital. Aparte de repartir la riqueza, algunos agentes sociales y políticos han subrayado la importancia de traer a los modelos de concentración también criterios para generarla.

Aunque no hayan sido creadas al servicio de ese objetivo ideológico, se han desarrollado herramientas estratégicas para la gestión de todas esas variables que hemos mencionado, como es, entre otros, el llamado RIS3. La Comisión Europea, siguiendo el criterio de especialización inteligente (smart specialization), puso en marcha la estrategia RIS3, basándose en que las políticas territoriales y económicas deben tomar en cuenta el contexto del territorio. En ese sentido, hay que situar a las estrategias de especialización inteligente dentro de la tendencia de «las nuevas políticas territoriales» o «nuevas políticas industriales». Es en estas nuevas políticas donde se trata la perspectiva desarrollada por Dani Rodrik en su trabajo «Política Industrial para el siglo XXI» (2004). Según esa perspectiva, la elección de las actividades económicas ha de decidirse a nivel comarcal, es decir, se reconoce la importancia de las estrategias de las comarcas (Aranguren et al., 2016). Además, la elección de las actividades económicas no depende únicamente del gobierno o del mercado, hay 
que crear nuevas formas de interacción entre el sector público y el privado para sacar adelante dicha tarea, y es eso, precisamente, lo que lo diferencia de las viejas políticas industriales. La gobernanza se ha convertido en una herramienta indispensable a la hora de tratar estos nuevos procesos.

\section{Gobernanza}

La gobernanza es un concepto con múltiples significados. Este concepto tan indefinido como esperanzador vendría a ser una nueva manera de gobernar alejada del control jerárquico del gobierno (Rhodes, 1997). Es un proceso basado en el trabajo colectivo y en la coordinación, con la intención de impulsar la integración de estrategias de agentes públicos y privados. Sin restar poder y competencias al gobierno, deja de lado el estilo jerárquico para tratar la gobernanza de un modo más cooperativo. Por lo tanto, impulsa condiciones institucionales que desarrollarán la capacidad de relizar cambios de mejora y actuación colectiva (Estensoro et al., 2011).

La gobernanza trata sobre los procesos gubernamentales realizados por un gobierno, mercado o red, que se materializa mediante la familia, tribu, asociación formal/informal o territorio y por medio de leyes, reglas, poder o lenguaje (Bevir, 2012). Trabaja maneras de gobernar que comportan la desaparición de los límites tanto internos como externos entre el sector privado y el público (Stoker, 1998). En ciencias políticas, la gobernanza expresa la transformación de la condición de estado de las democracias (Innerarity, 2011), pues esas democracias están abocadas a caminar de modelos jerárquicos y soberanos hacia modelos más cooperativistas. Por eso, la gobernanza vendría a ser la nueva manera de gobernar la sociedad.

Bevir y Rhodes (2010) diferencian tres fases al analizar la literatura de la gobernanza que se desarrolla en la administración pública. La primera fase, «la gobernanza en red», está relacionada con el patrimonio institucional de la reforma neoliberal del estado (Rhodes, 1997; Stoker, 1998; Subirats et al., 2002). El poder del estado está distribuido en una amplia variedad de redes espacial y funcionalmente diferentes, que engloba a todos los tipos de organizaciones públicas, privadas y de beneficiencia. Por lo tanto, hace referencia a la capacidad de autogobierno de las redes de agentes. La segunda fase sería la de «la metagobernanza», la cual se centra en el rol del estado, en "volver a traer al estado al primer plano» (bringing the state back in yet again) (Jessop, 2007: 54). En esta fase, el estado dirige y organiza las instituciones, gobiernos y grupos de redes, en vez de ofrecer los servicios directamente mediante las burocracias del estado. Además, al estado se le adjudica la responsabilidad de una mayor dirección política, ya que puede mostrarse a favor de los resultados de manera asimétrica (Jessop, 2007). 
Finalmente, Bevir y Rhodes (2010) tratan en la tercera fase el concepto de «gobernanza descentralizada». Según esta perspectiva las explicaciones narrativas son imprescindibles para investigar los procesos de gobernanza. Así, debemos comprender las creencias y las prácticas de los agentes en el contexto de las tradiciones particulares y respondiendo a dilemas específicos. Por lo tanto, debemos explicar los procesos de gobernanza partiendo de contextos, lugares o procesos sociales concretos y tratando siempre la perspectiva ascendente, es decir, centrada en los agentes.

En las estrategias de las especializaciones inteligentes la gobernanza juega un papel muy importante. Según esta perspectiva, lo que hay que trabajar no son «las estrategias gubernamentales», sino «las estrategias comarcales»; esto es, aquellas que identifican y consiguen inversiones dirigidas a prioridades tecnológicas, innovadoras y científicas. El gobierno efectuará esta tarea junto con agentes empresariales, intelectuales y la sociedad civil. Es decir, las decisiones no las tomará el gobierno por su cuenta, serán resultado de un proceso, concretamente del proceso que realizarán los agentes que participan en «hélices cuádruples». De este modo la comarca sería el conjunto de actores que se agrupa en una zona geográfica concreta (DFG, 2015:132); por lo tanto, el mismo proceso de gobernanza y la participación de los agentes deben situarse en el centro del proceso. Al fin y al cabo, en la estrategia RIS3 la gobernanza es un conjunto de procesos de coordinación basados en el diálogo, la participación y el compromiso, mediante el cual se desarrolla el proceso de participación del RIS3.

\section{RISS 3}

El RISS 3 es la base estratégica planteada por la línea principal de las políticas territoriales actuales de Europa. El concepto de especialización inteligente ha aportado una nueva perspectiva de la Política Comarcal de la Comisión Europea en el contexto de la Estrategia Europa 2020. El Consejo de la Unión Europea aprobó formalmente en diciembre del año 2013 las nuevas normas y legislación que regirán la siguiente ronda de inversión de las políticas de cohesión para el periodo 2014-2020. Estas son las claves de esa nueva perspectiva (UE, 2014):

\section{Convertir la innovación en prioridad para todas las comarcas}

Esta estrategia da prioridad a las inversiones en investigación, desarrollo e innovación a nivel comarcal. Estas prioridades deben sostener el cambio estratégico estructural en la economía de la comarca (Aranguren et al., 2016). Esta estrategia exige a los responsables políticos que tengan en cuenta la interrelación entre diferentes aspectos de crecimiento sostenible, sensato e integrador.

2. Centrarse en la inversión y crear sinergias

El RIS3 centra los esfuerzos e inversiones del desarrollo económico en los puntos fuertes de cada comarca, para así aprovechar las posibilidades económicas y las ten- 
dencias que están destacándose y tomar medidas para impulsar el crecimiento económico de cada comarca. El RIS3 pretende garantizar las sinergias entre las políticas europeas y la financiación europea.

\section{Mejorar el Proceso de Innovación}

El RIS3 exige alternativas estratégicas sensatas y una formulación de políticas basadas en la certeza. Las prioridades se establecen en el contexto del "proceso de descubrimiento activista». Este proceso hay que llevarlo a cabo al amparo de la inteligencia estratégica relacionada con los activos de las comarcas, es decir, debe tomar en cuenta las conexiones y los vínculos respecto a estructuras industriales, clústers, universidades, institutos de investigación, ciencia, tecnología, conocimientos, capital humano, medio ambiente, mercado, sistemas de gobernanza y otras comarcas. Además, debe tener en consideración también los retos de la comarca, el envejecimiento de la población, las incompatibilidades del mercado laboral, la ubicación lejana y los aspectos medioambientales. Al mismo tiempo debe desarrollar sus ventajas competitivas y su potencial de excelencia, entre otros, la previsión, la tendencia, el análisis de los puntos fuertes y débiles, las oportunidades y amenazas, la adjudicación tecnológica, el análisis de clúster y el conocimiento activista de los mercados.

\section{Mejorar la gobernanza}

El proceso RIS3 debe estar dirigido a las comarcas y basado en el acuerdo; por lo tanto, tiene que sustentarse en la interacción. El RIS3 anima a todos los participantes a unirse bajo una visión compartida. Su objetivo sería relacionar a las grandes, medianas y pequeñas empresas, impulsar la gobernanza a diferentes niveles y ayudar a generar capital creativo y social dentro de la comunidad.

Por lo tanto, el objetivo de la estrategia RIS3 sería «encontrar» las prioridades del desarrollo territorial mediante el diálogo entre diferentes tipos de conocimientos. Dichas prioridades no las establece únicamente el gobierno, también participarán en esa tarea agentes del campo de la ciencia y la tecnología, empresarios y, en general, la sociedad civil. Estos cuatro tipos de agentes compondrán la llamada «hélice cuádruple», y mediante plataformas adecuadas para facilitar el trabajo en equipo, se recopilará abundante conocimiento, desarrollándose así una estrategia de especialización inteligente. El resultado del proceso serían actividades de producción competitivas que un territorio debería tener en el futuro. Aunque la estrategia RIS3 se desarrolle como instrumento para fusionar la innovación en investigación y la cohesión social, al fin y al cabo su objetivo es la competitivad de los territorios, esta vez mediante la «diversificación especializada».

Esa horizontalidad teórica entre agentes no niega la necesidad de coordinar las redes de gobernanza, ni el diferente nivel de competencia y posición jerárquica que en esa coordinación tienen algunas instituciones. En el mismo sentido, las lógicas verticales serán necesarias a la hora de definir sectores prioritarios. Finalmente, las bases de la estrategia RIS3 serían la eficiencia inversora, el aprovechamiento de las 
virtudes de cada territorio, la apuesta por la investigación y conseguir la implicación de todos los grupos de interés, junto con la evaluación correspondiente de todo ello (Foray et al., 2009).

El punto de partida de las estrategias de especialización inteligente será el llamado "proceso de descubrimiento activista». En este proceso basado en los puntos fuertes del territorio deben participar conjuntamente: el sector público, el sector productivo, el sector del conocimiento y la sociedad civil (la hélice cuádruple), con el propósito de construir una estrategia territorial. En ese proceso es necesario configurar un modelo de gobernanza con múltiples niveles que recoja las aportaciones de los diferentes agentes y garantice la continuidad.

\section{Una mirada a las comarcas de Gipuzkoa}

La estrategia RIS3 establece los criterios para un desarrollo basado en el conocimiento y la especialización, mediante actividades innovadoras y de gran valor añadido. Eso exige definir las prioridades y necesidades fundamentales de una comarca, en aras de encauzar políticas e inversiones. Como ya hemos mencionado, la concreción y realización de esas prioridades la debe hacer la «hélice cuádruple», es decir, en el proceso tienen que participar los gobiernos, los agentes del conocimiento, las empresas y la sociedad civil. Este principio -la hélice cuádruple- organiza el nuevo modelo de gobernanza.

La Dirección de Desarrollo Territorial de Gipuzkoa, en colaboración con el grupo de investigación Parte Hartuz, pusieron en marcha en los años 2014-2015 el proyecto Gogoeta Taldea (grupo de reflexión), dentro de la iniciativa Gipuzkoa Sarean (Gipuzkoa en red), con el objetivo de ofrecer una visión externa a las estrategias de las comarcas de Gipuzkoa.

Imagen 1

Modelo de gobernanza de hélice cuádruple

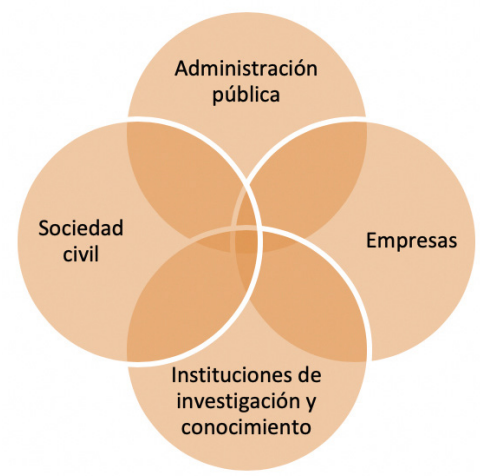

Fuente: elaboración propia 
Gipuzkoa Sarean es un proyecto de acción-investigación puesto en marcha en el año 2009 por la Diputación Foral de Gipuzkoa (DFG). La base de este proyecto es construir un nuevo modelo de relaciones que permita construir el desarrollo territorial de Gipuzkoa de una nueva manera. Para que esto se lleve a cabo, el proyecto accióninvestigación debe establecer estos objetivos principales (DFG, 2015):

1. Crear espacios para la colaboración entre responsables políticos y técnicos de las comarcas, la DFG y de otros niveles.

2. Desarrollar en los diferentes departamentos de la DFG modos que sean más participativos hacia las comarcas.

3. Conseguir un alineamiento entre las políticas de la DFG y los retos de las comarcas.

4. Fomentar en las comarcas modelos de relación participativos.

5. Fomentar en las comarcas el desarrollo de la visión y el pensamiento estratégico.

6. Acercar algunas instituciones que trabajan a nivel de Gipuzkoa (por ejemplo, universidades, centros tecnológicos, o Gipuzkoa Berritzen) y las realidades comarcales.

Como hemos mencionado, Gogoeta Taldea ha sido creado dentro del proyecto Gipuzkoa Sarean, y el grupo de trabajo está integrado por miembros de universidades de Gipuzkoa (Universidad de Mondragón, Universidad del País Vasco y Universidad de Deusto), centros tecnológicos (IK4 y Tecnalia) y Gipuzkoa Berritzen. Su objetivo principal es, por un lado, dar una visión externa y constructiva a los actores comarcales sobre sus procesos de desarrollo, y, por otro, acercar las universidades y centros tecnológicos a la realidad de las comarcas.

En este proceso impulsado por Gipuzkoa Sarean han participado principalmente técnicos, directores y políticos de agencias de desarrollo. Todos estos agentes que trabajan en la revisión de los modelos de desarrollo y relación comarcales de Gipuzkoa han reflexionado sobre cúal es el efecto de su actuación colaborativa sobre los retos de las comarcas, con el objeto de aprender de este proceso y, en su caso, abrir nuevos caminos. En definitiva, la aportación innovadora de Gogoeta Taldea ha sido la de unificar la visión de los agentes comarcales a la hora de diseñar las estrategias de las comarcas, y relacionarla con una visión externa.

Para llevar a cabo este proceso de reflexión emprendido en enero de 2014 se ha empleado el análisis de la estrategia territorial RIS3 impulsada por la Comisión Europea; en concreto, se han usado los 6 pasos propuestos para el establecimiento de la estrategia territorial: diagnóstico, gobernanza, visión compartida, prioridades, 
plan de acción y seguimiento, y finalmente, evaluación. Esta vez, pondremos el foco en el análisis del modo de funcionar y gobernar de las comarcas de Gipuzkoa, sus agencias de desarrollo y el gobierno, y expondremos una reflexión compartida sobre sus dificultades y sobre los principales retos que tiene actualmente.

\subsection{Una aproximación a la gobernanza}

En el caso de Gipuzkoa, no se ha identificado ningún espacio con el modelo de «hélice cuádruple» propuesto por la estrategia RIS3. Existen algunas mesas temáticas y foros de limitada actividad y composición, pero no se han detectado foros o espacios generales compuestos por la administración pública, empresas, instituciones del conocimiento y sociedad civil. Por lo tanto, podemos concluir que es necesario extender y reforzar una zona de intersección con el fin de desarrollar este modelo de gobernanza.

\subsubsection{La perspectiva técnica}

Los puntos fuertes identificados atendiendo a la perspectiva técnica tienen relación directa con los recursos humanos. Hay personas muy valiosas trabajando en el servicio técnico, con un gran nivel de compromiso, capacidad técnica y conocimiento de la realidad. Por lo tanto, no es de extrañar que haya estructuras de gobernanza formales maduras, sólidas y firmes.

En cambio, el punto débil lo situaríamos en el proceso de relación laboral de los recursos humanos. Se ha advertido la necesidad de implementar una relación y una coordinación entre los técnicos de las agencias y los técnicos municipales. Habría que reforzar la colaboración entre los técnicos de las agencias y los técnicos municipales, así como el conocimiento mutuo. Del mismo modo, habría que reforzar también la colaboración y la comunicación entre los representantes políticos municipales, los técnicos municipales y los gestores y técnicos de las agencias comarcales.

Para eso, podemos considerar como significativo y positivo el hecho de tener en cuenta la presencia y protagonismo de los técnicos en espacios integrados exclusivamente por políticos. Pero al mismo tiempo, debemos mencionar la necesidad de aumentarla. Por ejemplo, sería una idea interesante que los técnicos participaran en las comisiones para mejorar la interlocución y la coordinación que tienen entre ellos; de hecho, la cultura de trabajo vigente dificulta la creación de redes de gobernanza abiertas y democratizantes.

Los técnicos tienen mucho qué decir en la construcción de la red de gobernanza, pues les corresponde a ellos actuar de facilitadores en el proceso de definición y realización del modelo de desarrollo, en el caso de que haya compromiso de construirlo 
de manera compartida. Para ello, sería conveniente transformar los estilos de trabajo burocratizados y departamentalizados.

\subsubsection{La perspectiva política}

Tal y como es habitual en política, el modelo de gobernanza queda demasiado sujeto, también esta vez, a los plazos electorales. El consenso político básico es muchas veces frágil, y sin un acuerdo previo entre los partidos, las decisiones formales no garantizan el éxito en el desarrollo de las políticas públicas. Así pues, sería conveniente buscar el acuerdo político.

Para ello, es imprescindible acordar y trabajar la coordinación entre los representantes políticos de los municipios. En este caso, puede ser de ayuda el liderazgo, la implicación y la visión sobre la comarca de ciertos alcaldes. Pero del mismo modo, se advierte la necesidad de aclarar los roles, precisamente para evitar las dificultades de trabajar desde una perspectiva comarcal.

Se ha evidenciado la dificultad de trabajar la compatibilidad de los problemas municipales y las estrategias comarcales. Por lo tanto, habría que unir los problemas municipales (corto plazo) con las estrategias comarcales (largo plazo). Se ha advertido la necesidad de definir las estrategias a largo plazo.

En general, se puede afirmar que, observando las posibilidades intercomarcales, la colaboración con otras comarcas es débil. Para reforzarla, sería adecuado aumentar el conocimiento mutuo y las relaciones con respecto a agencias de desarrollo de otras comarcas. Deberían crearse condiciones para extender la mirada más allá de los límites comarcales y crear nuevas sinergias.

\subsubsection{Otros agentes}

$\mathrm{Al}$ analizar las relaciones existentes entre los agentes que participan en la estrategia de «hélice cuádruple» de gobernanza, se ha evidenciado la necesidad de reforzar los puentes con el tejido industrial y el sistema educativo. Deberían fortalecerse las relaciones entre los agentes, y para ello habría que estrechar lazos en la red $\mathrm{I}+\mathrm{D}+\mathrm{i}$.

Desde el ámbito de la sociedad civil, es evidente que su conocimiento es limitado. Aunque se hayan realizado presentaciones a agentes externos, a la gente le queda lejos la Agencia de Desarrollo. En muchas comarcas se entiende la mancomunidad como una agencia que presta servicios. Por lo tanto, sería de gran ayuda hacer una labor de socialización explicando que el desarrollo es más que la competitividad entre empresas, pudiendo ser esta una manera de aumentar la implicación de la población. Hay que crear condiciones para debatir y acordar el modelo de desarrollo comarcal junto con los agentes y ciudadanos, para así poder abrir la red de gobernanza e ir disminuyendo la distancia que se mencionaba antes. 
La tarea de crear una red de relaciones entre los diferentes agentes ya existe, pero a veces es más palpable que otras, y la mejor manera de fortalecer la colaboración y el conocimiento sería seguir dando pasos en esa dirección. Como hemos visto, los actores no tienen muchas oportunidades para estar juntos: los municipios grandes y pequeños, las pequeñas y grandes empresas, los agentes económicos y la sociedad civil, las instituciones/ciudadanos y los agentes estratégicos, etc. Por lo tanto, deberían crearse más espacios para el conocimiento mutuo de los agentes.

\subsection{Los retos de la gobernanza}

El principal reto lo situamos en la necesidad de una continua reflexión interna por parte de la Agencia de Desarrollo, especialmente en torno a dos temas fundamentales: el modelo de desarrollo comarcal y el propio rol de la Agencia. Al mismo tiempo, junto con esas reflexiones, la Diputación Foral de Gipuzkoa debería reconocer y apoyar el trabajo y el valor de las agencias.

Otro de los principales retos para garantizar la continuidad y desarrollo de la perspectiva estratégica comarcal sería reforzar los procesos y mecanismos que incrementan la participación de organismos no cívicos, sindicales ni gubernamentales.

Por otra parte, en lo que respecta a las infraestructuras del conominiento, sobre todo las de formación para el empleo, deberían coordinarse todos los agentes presentes, y trabajar desde una perspectiva de demanda. Del mismo modo, hay que potenciar los centros de formación profesional comarcales, facilitar la relación entre empresas y tecnología.

Así mismo, se quiere subrayar que, en muchos casos, las grandes empresas no están en la lógica de la Agencia, y que otras veces, en cambio, son las pequeñas las que estan alejadas. Por lo tanto, se debería priorizar una definición local, exacta, de lo que es la estrategia de la agencia de desarrollo: gestión coordinada de zonas industriales, servicios a empresas...

Como se ha apuntado anteriormente, la Agencia debería ser un espacio para facilitar la orientación estratégica de la comarca, y para ello habría que construir prioridades y liderazgos compartidos, ya que es imprescindible dar pasos para acordar la definición de las líneas de trabajo junto con los agentes comarcales (la hélice cuádruple). $\mathrm{Y}$ en esa orientación, por una parte, habría que potenciar la relación entre las agencias de desarrollo y los agentes políticos, pues es necesario reforzar la relación entre la representación política y la dirección de la Agencia. El consenso político garantizaría la sostenibilidad del proyecto. Y por otra parte, habría que revisar también el modelo de organización interna de la Agencia, para desarrollar el rol de facilitador y cultivar capacidades. 


\section{Conclusiones}

Sin infravalorar la difusión que puedan tener las referencias de carácter más participativo de la herramienta RIS3, en la cual se ha implicado la iniciativa Gipuzkoa Sarean, puede que haya que situar sus adaptaciones democráticas dentro de la profunda reflexión en torno a ciertos conceptos. La profundización de la estrategia RIS3 a nivel provincial debería situarse en el marco de un debate filosófico más profundo; es decir, en el debate sobre construcción de país y de estado contemporáneo a realizar por agentes económicos, sociales y políticos vascos. Es difícil concretar en estos momentos cuál puede ser el espacio idóneo para ello, pues el modelo de gobernanza es bastante complejo en nuestro territorio.

Las redes de gobernanza que ha habido hasta ahora han estado demasiado sujetas a las estructuras competenciales administrativas vigentes (Ayuntamientocomarca-Gipuzkoa-CAV-Estado), pero aunque no sea más que por una perspectiva de eficacia socio-económica, Gipuzkoa y sus comarcas deberían situarse dentro del desarrollo social del resto de provincias vascas, tomando en consideración la articulación territorial vasca. Aunque actualmente no existan espacios institucionales claros para desarrollar estrategias RIS3 que tengan en cuenta la territorialidad, puede haber herramientas adecuadas para esa tarea, como pueden ser, entre otros, Gaindegia, Eusko Ikaskuntza o las universidades vascas.

Las carencias de la estrategia RIS3 son el modelo de «mesa redonda» más determinantes. Por un lado, esta estrategia exige una profunda innovación a nivel empresarial, en el caso de que se pretenda cuidar una perspectiva integradora, es decir, una organización interna empresarial horizontal, la participación de cooperativas, etc. Al mismo tiempo, si se quieren desarrollar verdaderas estrategias territoriales dentro de la dinámica RIS3 hay que aumentar la implicación de las principales empresas. En muchos casos no ocurre así. Por otro lado, a nivel institucional tampoco está suficientemente desarrollada la coordinación entre diferentes estructuras, y suele ser difícil desarrollar visiones compartidas cuando aumenta la complejidad de las instituciones territoriales con poder de decisión. Por último, y este sería uno de los retos más importantes, hasta ahora en la estrategia RIS3 no se ha potenciado especialmente la participación de la sociedad civil -agentes sociales y ciudadanos de a pie-. Se le ha dado prioridad a reforzar las relaciones entre investigación/innovación y empresas, con la mediación de las instituciones. Del mismo modo, tampoco se han cuidado especialmente los sistemas de evaluación e inspección relacionados con la responsabilidad pública (accountability) (Navarro, 2013).

Dentro de la iniciativa Gipuzkoa Sarean, en los últimos años se han ejecutado diversas estrategias para resolver las carencias que se han mencionado, las cuales han ido estrechamente ligadas al diálogo entre innovación social e innovación tecnoló- 
gica. A pesar de ello, aunque se han dado pasos desde la perspectiva de «la definición democrática de las líneas de competitividad», todavía impera la lógica de mercado en la definición de la (re)generación del capital y de los principales ámbitos de desarrollo; y la especialización a nivel territorial se entiende ante todo en parámetros de mercado-competitividad.

Seguramente resultará difícil un cambio radical del modelo de desarrollo mediante las estrategias RIS3, pero aún no se ha llevado al límite toda la potencialidad democrática de dicha estrategia. Más allá del modelo teórico, todavía son muy evidentes los límites fácticos que tiene la «hélice cuádruple».

Para terminar, todas estas reflexiones sobre el territorio habría que situarlos en torno al eje transversal y vertical: el ciudadano, y todas las organizaciones sociales ciudadanas, sean empresas, sindicatos, movimientos sociales, organizaciones no gubernamentales o asociaciones privadas de otro tipo. Cualquier orientación relacionada al desarrollo territorial/comarcal debería estar no sólo al servicio de la ciudadanía, sino también sujeta a su arbitrio. Ese sería el imprescindible requisito de la democratización: todas las decisiones, desde la iniciativa hasta la evaluación más allá de la gestión, deberían llevarse a cabo de manera compartida.

¿En qué consiste la especialización inteligente de Euskal Herria? ¿En un desarrollo endógeno de la cultura vasca que garantice un desarrollo humano sostenible, o en un tipo de especialización que habra paso a la disneyficación? ¿Algo que se base especialmente en los servicios, o una especialización tecno-industrial que garantice el desarrollo social interno? ¿Un modo de creación de renta basado en el respeto a la tierra, o un modelo que exija la explotación de recursos propios y de otros pueblos y que aumente la deuda ecológica? Está claro que un desarrollo territorial justo debe fusionar el desarrollo social y humano y el desarrollo económico e institucional de una manera sostenible (Alburquerque, 2008); pero las elecciones concretas están en nuestras manos, en manos de la ciudadanía.

\section{Bibliografía}

ALBURQUERQUE, Francisco; COSTAMAGN, Pablo y Carlo FERRARO. (2008). Desarrollo local, descentralización y democracia. Ideas para un cambio. Buenos Aires: UNSAM.

ARANGUREN, María José; MORGAN, Kevin y James, WILSON. (2016). Implementar la RIS3. El caso del País Vasco. Bilbao: Orkestra Instituto Vasco de Competitividad.

BEVIR, Mark y R.A.W. RHODES. (2010) The State as Cultural Practice. Oxford: Oxford University Press.

BEVIR, Mark (2012). Governance: A very short introduction, Oxford: OUP Oxford. 
CASTELLS, Manuel (2000) The rise of the network society, Oxford: Blackwell.

COMISIÓN EUROPEA (2014) Estrategias nacionales y regionales para la especialización inteligente (RIS3), http://ec.europa.eu/regional_policy/sources/ docgener/informat/2014/smart_specialisation_es.pdf (consulta: 29-10-2017).

DIPUTACIÓN FORAL DE GIPUZKOA, ORKESTRA INSTITUTO VASCO DE COMPETITIVIDAD (2015) Gipuzkoa sarean Bidean, Donostia: Diputación Foral de Gipuzkoa.

ESTENSORO, Miren; LARREA, Miren y Mikel ZURBANO. (2011) «Tokiko ekonomia-gobernantza berrikuntza sozialaren begiradatik». Uztaro: giza eta gizarte-zientzien aldizkaria, 77, 23-41.

FORAY, D.; DAVID, P.A. y B. HALL. (2009). «Smart Specialisation - The Concept» Knowledge Economists Policy Brief no. 9. http://ec.europa.eu/invest-in-research/ pdf/download_en/kfg_policy_brief_no9.pdf?11111.

INNERARITY, Daniel. (2011). «¿Qué es eso de la gobernanza?». Tiempo de paz, 100, 228-233.

JESSOP, Bob. (2007). State Power: A Strategic-Relational Approach. Cambridge: Polity Press.

JESSOP, Bob. (2006). «Spatial fixes, temporal fixes and spatio-temporal fixes». En KARLSEN, James y Miren LARREA. (2015). Desarrollo territorial e investigación acción. Innovación a través del diálogo. Bilbao: Publicaciones Universidad de Deusto.

NAVARRO, Mikel y Edurne MAGRO. (2013). "Complejidad y coordinación en las estrategias territoriales. Reflexiones desde el caso vasco». Ekonomiaz, 82, Gobierno Vasco, Gasteiz.

RHODES, R. A. W. (1997). Understanding Governance. London: Open University Press.

RODRIK, Dani. (2004). Industrial policy for the twenty-first century. Cambridge, Massachusetts: Harvard University.

STOKER, Gerry. (1998). «Governance as theory: five propositions», International Social Sciences Journal, 50 , 17-28. https://doi.org/10.1111/1468-2451.00106.

SUBIRATS, Joan (Coord.) (2002). Redes, territorios y gobierno : nuevas respuestas locales a los retos de la globalización. Barcelona: Diputación de Barcelona.

TILLY, Charles. (2004). Contention and democracy in Europe, 1650-2000. New York: Cambridge University Press.

ZUBIAGA, Mario. (2012). «Democratization and Contentious Politics: Basque Statebuilding as Collective Action». En: ZUBIAGA, Mario. (2012) (ed). Towards a Basque State: Nation Building and Institutions. Bilbao: IparHegoa Fundazioa. 
